\title{
Gas Hydrate on Continental Margins
}

\author{
Umberta Tinivella, ${ }^{1}$ Michela Giustiniani, ${ }^{1}$ Xuewei Liu, ${ }^{2}$ and Ingo Pecher ${ }^{3}$ \\ ${ }^{1}$ Istituto Nazionale di Oceanografia e di Geofisica Sperimentale, OGS, Borgo Grotta Gigante 42/C, 34010 Trieste, Italy \\ ${ }^{2}$ Institute of Geophysics and Geomatics, China University of Geosciences, 29 Xueyuan Road, Beijing 100083, China \\ ${ }^{3}$ GNS Science, 1 Fairway Drive, P.O. Box 30368, Lower Hutt 5010, New Zealand \\ Correspondence should be addressed to Umberta Tinivella, utinivella@ogs.trieste.it
}

Received 23 November 2011; Accepted 23 November 2011

Copyright (C) 2012 Umberta Tinivella et al. This is an open access article distributed under the Creative Commons Attribution License, which permits unrestricted use, distribution, and reproduction in any medium, provided the original work is properly cited.

Gas hydrate is a solid crystalline material composed of water and natural gas that forms at low temperature and high pressure. Gas hydrates represent an important reservoir of natural gas, even if the current global estimate is very rough. The increasing attention to gas hydrates is arising from (1) the assessment of methane hydrates as a new relatively clean energy source, (2) the relationship between gas hydrate and global climate, (3) the geological hazards related to gas hydrates, and (4) recent programs to sequester $\mathrm{CO}_{2}$ in gas hydrate reservoirs.

Generally, gas hydrate deposits are investigated by using several geophysical methods. The seismic technique, which is the most used, allows detecting an indicator of the hydrate and free gas accumulations, known as bottom simulating reflector (BSR). Moreover, seismic data provide information about the geometry of the main geological structures, allowing possible explanations of the presence/absence of gas hydrate on continental margins. In the last years, the scientific community has started to integrate geophysical, geochemical, and heat-flow data in order to detect and characterize gas hydrate and free gas volumes and their distribution in marine sediments. Thus, reviews of extensive geophysical surveys and direct measurements combined with geological interpretation and theoretical modelling will increase understanding on the occurrence, distribution, and concentration of gas hydrate and the underlying free gas beneath the ocean bottom, as demonstrated by the papers included in this issue.

The main topic of three of the papers is modelling. L. Huaishan et al. established sound models with hydrate and free gas in the water and sediment in order to extract information about gas bubble size, gas content, sound velocity, attenuation, resonance frequency, and scattering cross-section from acoustic data. J. Majorowicz et al. studied the dynamic permafrost and gas hydrate history in the Northern Canadian margin using a numerical model to illustrate how changes in setting and environment, especially periodic glacial ice cover, affected gas hydrate stability. The models show that gas hydrates have persisted through interglacial episodes. The paper of V. Tinivella et al. is focussing on the relationship between gas hydrates and slope instability in polar regions. Their modelling suggests that, during the interglacial period, gas hydrate influences slope stability and climate change only at the beginning of the warming period and only if the sea bottom is affected by temperature variations.

Another part of this issue is dedicated to several geophysical methods applied to study gas hydrate reservoirs. D. Chen et al. analysed 3D seismic data to identify polygonal faults in the South China Sea. They observed that gas hydrates are located in the sediments above a polygonal fault layer. Polygonal faults can act as pathways for the migration of fluids, which can supply hydrocarbons for the formation of gas hydrates. To study physical properties of methane gas hydrate-bearing sediments, it is necessary to synthesize laboratory samples due to the limited availability of cores from natural deposits, as reported by E. V. L. Rees et al. They performed X-ray computed tomography during hydrate formation and decomposition phases. The investigation revealed the impact of water saturation on location and morphology of hydrate in both laboratory and natural sediments during repeated hydrate formations. Significant redistribution of hydrate and water in the samples was 
observed over both the short-term and long-term. V. D. Solovyov et al. used short-pulsed electromagnetic field and vertical electric-resonance sounding, integrated with special technology of satellite data processing and interpretation, offshore Antarctic Peninsula. J. Tomasini et al., I. VargasCordero et al., and X. Wang et al. analysed 2D seismic reflection data to determine gas hydrates and associated free gas distribution offshore Uruguay, offshore Southern Chilean margin, and in the South China sea, respectively, showing a great potential for this nonconventional resource and encouraging further research. In addition, two papers, by $\mathrm{D}$. Wu et al. and $\mathrm{N}$. Wu et al., are dedicated to geochemical analysis of samples acquired in the South China Sea, confirming seismic results that suggest the potential for gas hydrate resources in this area.

The multidisciplinary of gas hydrate research is emphasized by the papers by S. Conti and D. Fontana, which focused on contribution of paleogas hydrate destabilization in the Miocene in the Northern Apennines, and C. Perissoratis et al., which reported a detailed multibeam survey and a gravity coring carried out in the Anaximander Mountains, Eastern Mediterranean, detecting an active gas hydratebearing mud volcano named Thessaloniki.

Because carbon dioxide also forms gas hydrates, methane hydrate studies have recently been associated with $\mathrm{CO}_{2}$ sequestration, which is the topic of other two papers. In these papers, approaches are analyzed for combining natural gas production from methane hydrate deposits and $\mathrm{CO}_{2}$ sequestration. G. Janicki et al. modelled two scenarios: the depressurization of an area $1,000 \mathrm{~m}$ in diameter and a one/two-well scenario with $\mathrm{CO}_{2}$ injection. The performed simulations show that depressurization with or without simultaneous $\mathrm{CO}_{2}$ injection is a practicable way to produce methane from subsea hydrate fields with assured rates over many years. A. Nago and A. Nieto reported the state-of-the-art of the $\mathrm{CO}_{2}$ hydrate and investigated the molecular exchange between $\mathrm{CO}_{2}$ and $\mathrm{CH}_{4}$ that occurs when methane hydrates are exposed to $\mathrm{CO}_{2}$, thus generating the release of natural gas while trapping carbon dioxide as a gas hydrate. Their work included laboratory studies on the synthesis, thermodynamics, phase equilibrium, kinetics, cage occupancy, and the methane recovery potential of the mixed $\mathrm{CO}_{2}-\mathrm{CH}_{4}$ hydrate, including both experimental and numerical data.

In conclusion, the fifteen papers reported here constitute a significant contribution to our knowledge on natural gas hydrates by presenting results from direct and indirect measurements on several continental margins, studying relationships between geophysical response and hydrate occurrence, and investigating geological aspects related to the gas hydrate presence. The papers also set the stage for future work to increase our knowledge on gas hydrates.

Umberta Tinivella Michela Giustiniani Xuewei Liu Ingo Pecher 

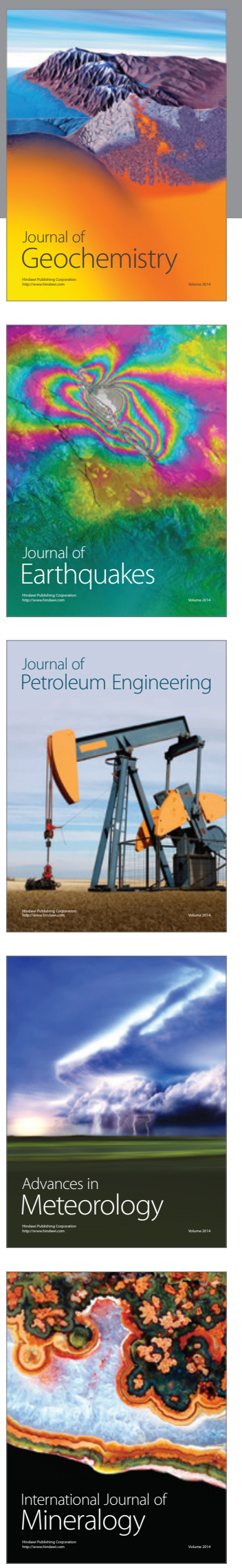
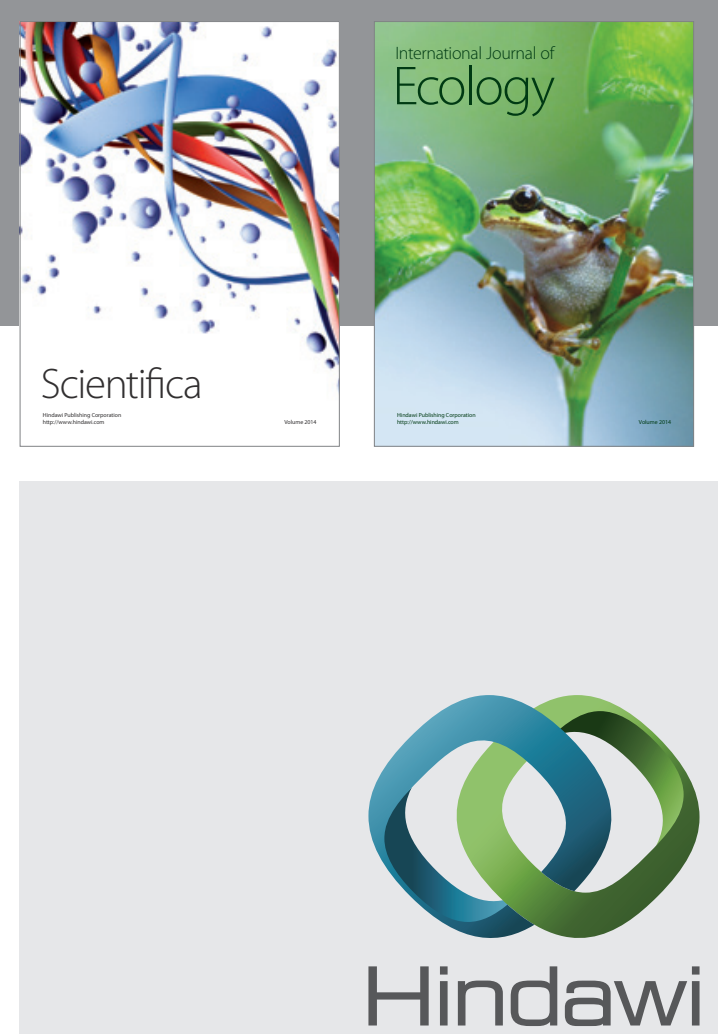

Submit your manuscripts at http://www.hindawi.com
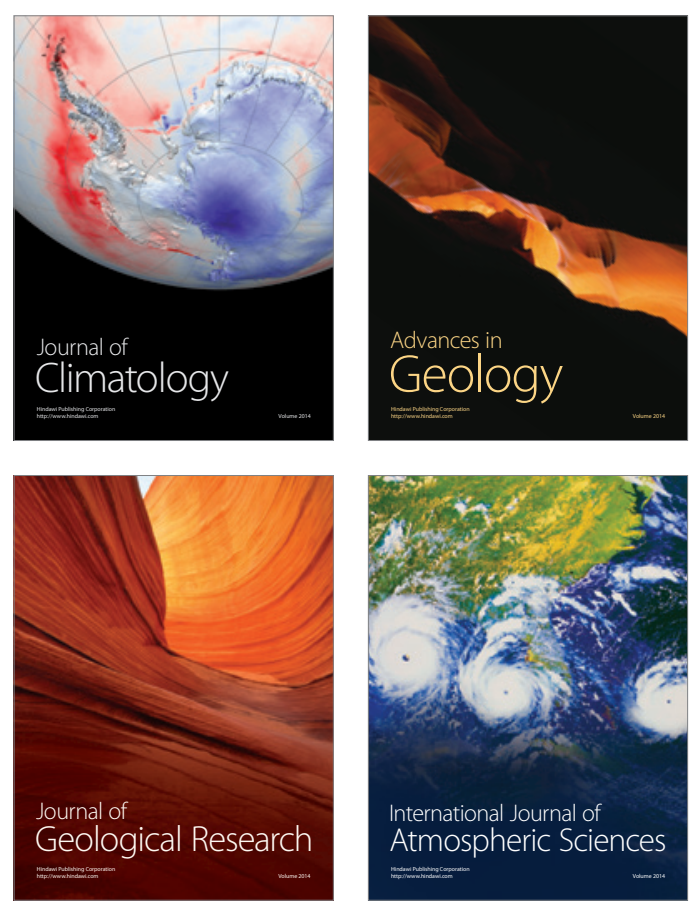
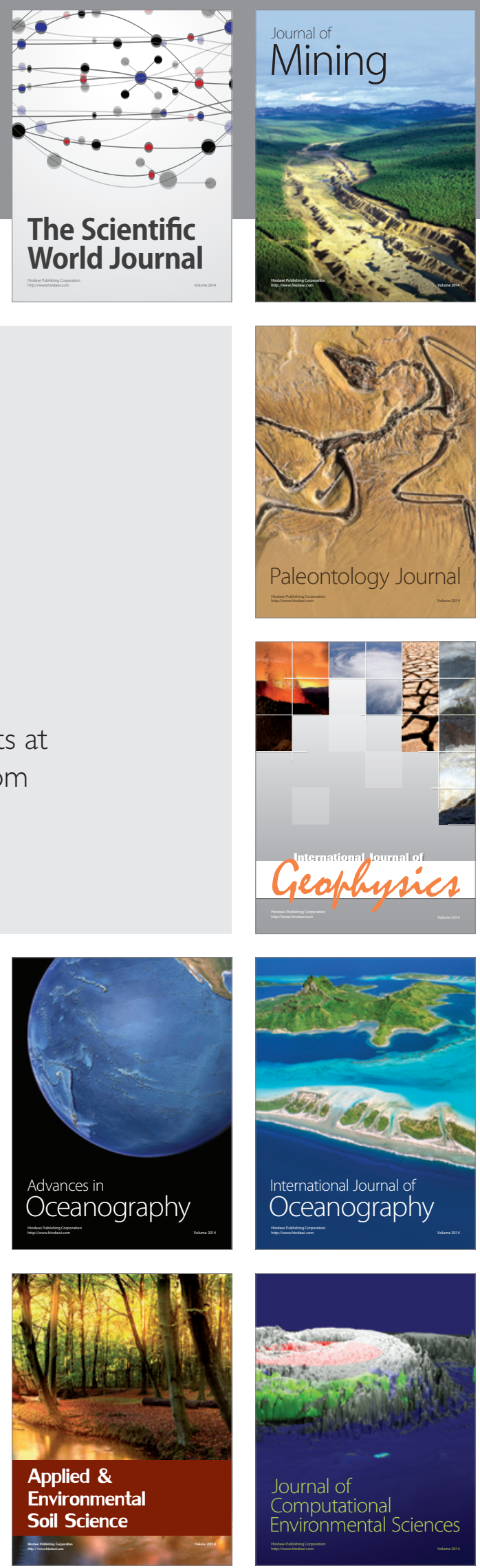